\title{
Time dictates: emerging clinical analyses of the impact of circadian rhythms on diagnosis, prognosis and treatment of disease
}

\author{
Authors: Andras D Nagy ${ }^{A}$ and Akhilesh B Reddy ${ }^{B}$
}

Since the advent of modern molecular tools, researchers have extensively shown that essential cellular machineries have robust circadian (roughly 24 hours) variations in their pace. This molecular rhythmicity translates directly into time-of-day-dependent variation in physiology in most organ systems, which in turn provides the mechanistic rationale for why timing on a daily basis should matter in many aspects of human health. However, these basic science findings have been slow to move from bench to bedside because clinical studies are still lacking to demonstrate the importance of timing. Therefore, it has not been clear how physicians should incorporate knowledge of natural 24 -hour rhythms into routine practice. This review is a brief summary of results from recently completed clinical studies on hypertension, myocardial infarction, diabetes mellitus, and adrenal dysfunction that highlights new evidence for the emerging importance of circadian rhythms in diagnosis, prognosis and treatment of disease.

\section{Introduction}

Twenty-four hour biological clocks - ie circadian clocks within living organisms are fascinating achievements of nature. They allow temporal separation of otherwise conflicting biochemical events by separating the physiological activities of cells into different stages of the day. ${ }^{1}$ Virtually all aspects of cellular and tissue biology oscillate daily, with huge changes in gene and protein expression affecting second messenger availability, membrane excitability, metabolic parameters, redox balance and antioxidant capacity. ${ }^{1}$ Many of these fundamental cellular properties are dysregulated when leading morbidities, such as cardiovascular and metabolic diseases, develop. Restoration of circadian rhythms as a means of treating such conditions has been demonstrated in animal models. ${ }^{2}$ However, large clinical trials have only recently been

Authors: Alecturer, Department of Anatomy, University of Pécs Medical School, Pécs, Hungary, and Marie-Curie IntraEuropean research fellow, University of Cambridge, Cambridge, UK; ${ }^{B}$ Wellcome Trust senior fellow, Department of Clinical Neurosciences, University of Cambridge, Cambridge, UK done to present clear evidence for physicians that timing matters at the bedside for common conditions. Nonetheless, delivering certain drugs at particular times of day to maximise their potency and minimise side effects has shown promise in patients. This is exemplified by the longstanding use of statins at night time, ${ }^{3}$ when their effects are maximal, and the use of specialised rhythmic dosing regimens that take into account endogenous 24 -hour rhythms in patients with cancer. ${ }^{4}$ Here we present some recent examples of cardiovascular, metabolic and endocrine pathophysiology that can be modulated with knowledge of the circadian clockwork.

\section{Hypertension}

Systolic and diastolic blood pressure in healthy normotensive individuals rapidly rise upon waking and decrease relative to daytime levels by $>10 \%$ during sleep. ${ }^{5}$ This pattern originates from daily rhythms in the renin-angiotensin-aldosterone system, autonomic activity, and rest- and activity-related differences in mental and physical stress. ${ }^{5,6}$ Diseases can alter sleep, autonomic and neuroendocrine function, with consequential modification of the 24-hour blood pressure profile. Such subtle alterations are associated with an increased risk of left ventricular remodelling, renal pathology, cardiovascular and cerebrovascular events and total mortality ${ }^{7}$.

The ABC-H study, ${ }^{8}$ a systematic review of nine hypertensive cohorts from Europe, Brazil and Japan $(n=13,844)$, showed that elevated night-time systolic blood pressure independently predicted higher rates of cerebrovascular events in most cohorts, and, overall, night-time systolic blood pressure independently predicted cerebrovascular events. In view of these results and in accordance with 2013 European guidelines for ambulatory blood pressure monitoring, ${ }^{9}$ there is no evidence-based rationale for the omission of routine monitoring of night-time systolic blood pressure in patients diagnosed with hypertension, since it has a key role in hypertension-related morbidity and mortality. Furthermore, in patients with acute stroke, each $10 \mathrm{mmHg}$ preserved dayto-night decline in systolic blood pressure seen in the 24-h blood pressure monitoring upon admission was associated with a two-fold increase in likelihood of complete functional recovery examined in univariate associations with neurological 
outcomes after 7 days of admission (odds ratio (OR) 2.24; 95\% confidence interval (CI) $1.16-4.32 ; \mathrm{p}=0.016$ ). If considering diastolic blood pressure the OR for complete recovery associated with each $10 \mathrm{mmHg}$ day-to-night decline was 4.63 (95\% CI 1.57-13.7; $\mathrm{p}=0.01) .^{10,11}$

Although daytime hypertension should not be ignored, night-time blood pressure might be the most important target for antihypertensive therapy in general. ${ }^{8,12}$ This conclusion of the ABC-H study is consistent with an open-label, singlecentre, randomised trial in which reductions in cerebrovascular events and cardiovascular mortality were greater when anti-hypertensives were given in the evening than in the morning. ${ }^{13}$ After a median follow up of 5.6 years, subjects ingesting $\geq 1$ BP-lowering medications at bedtime exhibited lower relative risk of total cerebrovascular events than those ingesting all medications upon awakening $(0.39$ (0.29-0.51); number of events 187 vs $68 ; \mathrm{p}<0.001)$. The difference between the treatment-time groups in the relative risk of major events (including cardio- and cerebrovascular death, myocardial infarction, ischemic stroke and hemorrhagic stroke) was also apparent ( 0.33 (0.19-0.55); number of events: 55 vs 18 ; $\mathrm{p}<0.001)$. Further randomised trials are urgently needed to provide a firm evidence base for the treatment of this prevalent condition.

\section{Myocardial infarction}

Daily rhythms in the physiological regulation of blood pressure and blood clotting underlie the well known increased incidence of acute ST-elevation myocardial infarction (STEMI) in the morning. ${ }^{5,6}$ However, whether the myocardium's ability to cope with ischaemia is subject to a clinically significant circadian pattern or onset time is a prognostic factor for outcomes has not been clearly defined. The largest clinical study so far to investigate rigorously these questions enrolled approximately

\section{Key points}

Night-time blood pressure might be the most important target of antihypertensive therapy.

The daily rhythm in the myocardium's vulnerability to ischaemia defines worse prognosis if ischaemia onset time is at night.

The primary target of treatment in type 2 diabetes mellitus should be to re-establish near-normoglycaemia before and after breakfast.

Conventional glucocorticoid replacement strategies result in serum cortisol profiles that little resemble the physiological 24 hour pattern, which is thought to be why outcomes are worse in patients with adrenal insufficiency and poor quality of life in rheumatoid arthritis.

KEYWORDS: Circadian rhythm, NSBP, ischaemia onset time, dawn phenomenon and near-physiological hormone replacement
24,000 patients with acute STEMI from a Swiss prospective multicentre registry. ${ }^{14}$ As a measure of ischaemic burden, circadian variation of peak creatine kinase concentrations and in-hospital mortality were assessed in a well defined population of patients $(n=6,223)$ who were treated with primary percutaneous coronary intervention less than 6 hours after symptom onset. Using harmonic regression methods, a 24-hour pattern in ischaemic burden was consistently identified in various subgroups of individuals. STEMI patients undergoing primary percutaneous coronary intervention whose symptom onset at night presented with the highest peak creatine kinase concentrations, whereas the lowest values were noted in patients with symptoms starting in the morning. Furthermore, the probability of in-hospital death was highest for patients with symptom onset around midnight $(\mathrm{p}=0.03)$. The rhythmic pattern in ischaemic burden was even more pronounced in patients with previous low risk of mortality. Thus, there is compelling evidence that the rhythmic nature of the myocardium's vulnerability to ischaemia defines the worse prognosis of STEMIs at night. Importantly, this pattern was independent of duration of ischaemia, raising the notion that symptom onset time might be a better factor for prognostic prediction than duration of ischaemia in STEMI patients undergoing primary percutaneous coronary interventions.

\section{Diabetes mellitus}

Overnight measurements in non-diabetic people suggests that both blood glucose and plasma insulin concentrations remain almost constant. ${ }^{15}$ Conversely, in patients with diabetes, a substantial increase in blood glucose before breakfast (ie fasting hyperglycaemia) is a frequent event known as the dawn phenomenon. ${ }^{15}$ In type 1 diabetes, the most likely explanation is that the effects of nocturnal secretion of growth hormone on blood glucose are not counterbalanced as a result of the absence of insulin. Treatment of type 1 diabetes is restricted to insulin replacement, necessitating consideration of the circadian rhythm of insulin sensitivity, which peaks from 12am until $3 \mathrm{am}$. When targeting fasting normoglycaemia at dawn, the risk of nocturnal hypoglycaemia could be effectively reduced with more physiological replacement of basal insulin by using continuous subcutaneous insulin infusion or long-acting insulin analogues, which do not result in peaks and troughs during the day ${ }^{15}$.

In type 2 diabetes, the dawn phenomenon has been attributed to a transient increase in hepatic gluconeogenesis at dawn in the absence of compensatory insulin secretion. ${ }^{15}$ In a study of 248 patients with type 2 diabetes, researchers precisely quantified the magnitude of the dawn phenomenon and its effect on overall control of blood glucose by measuring overnight interstitial glucose concentrations with continuous ambulatory monitoring. ${ }^{16}$ An increase in interstitial glucose (13-20 mg/dL) from the nocturnal nadir to pre-breakfast values was reported in all treatment groups, the patients in which took only oral therapies or were diet-controlled. In all diabetic groups investigated in the study, pre-breakfast mean glucose concentrations (ie the value after the overnight fasting) were significantly higher than the nadir values observed during the nocturnal periods $(\mathrm{p}<0.0001)$. The magnitude of group dependent increases gives a range of $13-20 \mathrm{mg} / \mathrm{dL}$ increase in interstitial glucose considered as the dawn phenomenon. 
Importantly, the dawn phenomenon had an effect on mean daily interstitial glucose and $\mathrm{HbA}_{1 \mathrm{c}}$ concentrations, which was independent of treatment. The impact of the dawn phenomenon on diabetic control in the overall population an increase of $12 \mathrm{mg} / \mathrm{dL}$ in mean glucose concentration and $4 \mathrm{mmol} / \mathrm{mol}$ [0.4\%] in $\mathrm{HbA}_{1 \mathrm{c}}$ - was in agreement with the $\mathrm{A}_{1 \mathrm{C}}$-Derived Average Glucose Study (ie an increment of 29 $\mathrm{mg} / \mathrm{dL}$ in glucose corresponds to a $1 \%$ increment in $\left.\mathrm{HbA}_{1 \mathrm{c}}\right) .^{17}$ Furthermore, the pre-breakfast increase in interstitial glucose extended to the post-breakfast period, when the highest concentrations of the day were noted (mean 191-208 mg/dL). ${ }^{16}$

Because these early morning hyperglycaemic episodes are thought to progressively impair pancreatic $\beta$-cell function in the context of insulin resistance mainly at dawn and independently of age, the primary aim of treatment in type 2 diabetes is to re-establish near-normoglycaemia before and after breakfast (ie to treat the dawn phenomenon). ${ }^{15,18}$ Furthermore, data from participants treated with diet alone showed that the dawn phenomenon is already present in those who are not on pharmacological treatment ${ }^{16}$ suggesting that treatment of the dawn phenomenon should be initiated before $\mathrm{HbA}_{1 \mathrm{c}}$ has increased beyond $7.0 \%$ rather than after, which is current practice. ${ }^{15}$ Evening replacement of basal insulin in type 2 diabetes, which avoids the dawn phenomenon effectively by restraining hepatic glucose production and lipolysis is an optimal treatment (effective, easy, safe and natural) that mimics the physiology of glucose homeostasis of healthy nondiabetic people. ${ }^{15}$

\section{Adrenal insufficiency}

Serum cortisol concentrations of healthy individuals show a robust peak before waking and fall to a nadir during night-time sleep. ${ }^{19}$ Hormone replacement therapy in adrenal insufficiency is a life-saving treatment. The total daily dose of hydrocortisone is divided and administered 2-3 times a day (Fig 1). However, the resulting serum cortisol profile does not closely resemble the physiological circadian pattern because sharp peaks, followed by rapid declines in cortisol concentrations cause overexposure followed by underexposure around the clock. ${ }^{19}$ Furthermore, the timing of the dose is likely to be different on a day-to-day basis, resulting in different temporal exposure profiles. Importantly, over-replacement of cortisol (particularly during night-time sleep) can lead to impaired glucose tolerance, obesity, osteoporosis, and sleep disturbances. ${ }^{19}$ Conversely, under-replacement during the daytime can be life threatening if the patient develops an intercurrent illness. ${ }^{19}$

In line with these assumptions, patients taking chronic adrenal hormone replacement live significantly shorter and report worse quality of life than do healthy controls. ${ }^{20,21} \mathrm{An}$ international survey of 1,245 patients with adrenal insufficiency showed that $38 \%$ of patients report difficulties with multiple dosing, greater fatigue, and greater impact on their quality of life. ${ }^{21}$ In small, uncontrolled studies of patients with adrenal insufficiency, infusion of hydrocortisone by a programmable pump has restored a normal cortisol circadian rhythm (Fig 1) and improved quality of life in most patients, although outcomes have not been systematically reported. ${ }^{22,23}$ Therefore, it is highly likely that the non-physiological daily profile of standard replacement therapy is a major contributor to worse outcomes in patients with adrenal insufficiency, although the

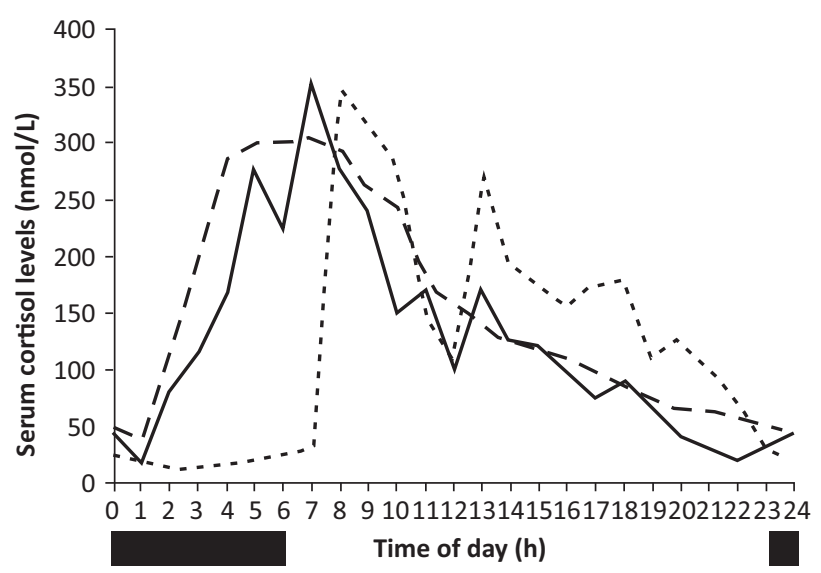

Fig 1. Mean 24-hour cortisol profiles in healthy people and patients with adrenal insufficiency. Solid = healthy people; dotted = adrenal insufficiency treated with conventional therapy; dashed = adrenal insufficient treated with programme infusion therapy. Dark bars represent periods of darkness. Adapted from Merza et al. ${ }^{23}$

level of evidence for this hypothesis is still poor because of a lack of randomised, placebo-controlled studies. Time-released oral formulations of corticosteroids have been introduced successfully for the treatment of rheumatoid arthritis. ${ }^{24}$ Similar approaches to create a more physiological daily profile for steroids in the circulation could be beneficial for treating related conditions, including adrenal insufficiency.

\section{Summary}

These recent clinical studies have demonstrated that circadian rhythms in human physiology offer true potential for physicians and clinical researchers to develop novel approaches in healthcare with more promising outcomes than conventional strategies. Furthermore, existing drugs and therapies, whose side-effect profiles are known well, can be used or repurposed in a more efficient manner, which therefore reduces development costs and alleviates potential safety concerns major hurdles during development of completely new drugs.

\section{References}

1 Rey G, Reddy AB. Connecting cellular metabolism to circadian clocks. Trends Cell Biol 2013; 23:234-41.

2 Dallmann R, Brown SA, Gachon F. Chronopharmacology: new insights and therapeutic implications. Annu Rev Pharmacol Toxicol 2014;54:339-61.

3 Knopp RH. Drug treatment of lipid disorders. N Engl J Med 1999;341:498-511.

4 Lévi F, Okyar A, Dulong S et al. Circadian timing in cancer treatments. Annu Rev Pharmacol Toxicol 2010;50:377-421.

5 Takeda N, Maemura K. Circadian clock and cardiovascular disease. J Cardiol 2011;57:249-56.

6 Smolensky MH, Portaluppi F, Manfredini R et al. Diurnal and twenty-four hour patterning of human diseases: acute and chronic common and uncommon medical conditions. Sleep Med Rev 2015;21:3-11.

7 Verdecchia P, Porcellati C, Schillaci G et al. Ambulatory blood pressure: an independent predictor of prognosis in essential hypertension. Hypertension 1994;24:793-801. 
8 Roush GC, Fagard RH, Salles GF et al. The Ambulatory Blood Pressure Collaboration in Patients Diagnosed with Hypertension $(\mathrm{ABC}-\mathrm{H})$ investigators. Prognostic impact from clinic, daytime, and nighttime systolic blood pressure in nine cohorts of 13,844 patients with hypertension. J Hypertens 2014;32:2332-40.

9 O'Brien E, Parati G, Stergiou G et al; on behalf of the European Society of Hypertension Working Group on Blood Pressure Monitoring. European Society of Hypertension position paper on ambulatory blood pressure monitoring. J Hypertens 2013;31:173168.

10 Bhalla A, Wolfe CD, Rudd AG. The effect of $24 \mathrm{~h}$ blood pressure levels on early neurological recovery after stroke. J Intern Med 2001;250:121-30.

11 Wallace DM, Ramos AR, Rundek T. Sleep disorders and stroke. Int J Stroke 2012;7:231-42.

12 Roush GC, Fapohunda J, Kostis JB. Evening dosing of antihypertensive therapy to reduce cardiovascular events: a third type of evidence based on a systematic review and meta-analysis of randomized trials. J Clin Hypertens (Greenwich) 2014;16:561-8.

13 Hermida RC, Ayala DE, Mojon A, Fernandez JR. Influence of circadian time of hypertension treatment on cardiovascular risk: results of the MAPEC study. Chronobiol Int 2010;27:1629-51.

14 Fournier S, Taffé P, Radovanovic D et al. Myocardial infarct size and mortality depend on the time of day - a large multicenter study. PLOS ONE 2015;10:e0119157.

15 Porcellati F, Lucidi P, Bolli GB, Fanelli CG. Thirty years of research on the dawn phenomenon: lessons to optimize blood glucose control in diabetes. Diabetes Care 2013;36:3860-2.

16 Monnier L, Colette C, Dejager S, Owens D. Magnitude of the dawn phenomenon and its impact on the overall glucose exposure in type 2 diabetes: is this of concern? Diabetes Care 2013;36:4057-62.

17 Nathan DM, Kuenen J, Borg R et al. Alc-Derived Average Glucose (ADAG) Study Group. Translating the A1C assay into estimated average glucose values. Diabetes Care 2008;31:1473-8.
18 Inzucchi SE, Bergenstal RM, Buse JB et al. Management of hyperglycemia in type 2 diabetes: a patient-centered approach: position statement of the American Diabetes Association (ADA) and the European Association for the Study of Diabetes (EASD). Diabetes Care 2012;35:1364-79.

19 Grossman A, Johannsson G, Quinkler M, Zelissen P. Therapy of endocrine disease: perspectives on the management of adrenal insufficiency: clinical insights from across Europe. Eur J Endocrinol 2013;169:R165-75.

20 Bergthorsdottir R, Leonsson-Zachrisson M, Oden A, Johannsson G. Premature mortality in patients with Addison's disease: a population-based study. J Clin Endocrinol Metab 2006;91:4849-53.

21 Forss M, Batcheller G, Skrtic S, Johannsson G. Current practice of glucocorticoid replacement therapy and patient-perceived health outcomes in adrenal insufficiency - a worldwide patient survey. BMC Endocrine Disorders 2012;12:8.

22 Løvås K, Husebye ES. Continuous subcutaneous hydrocortisone infusion in Addison's disease. Eur J Endocrinol 2007;157:109-12.

23 Merza Z, Rostami-Hodjegan A, Memmott A et al. Circadian hydrocortisone infusions in patients with adrenal insufficiency and congenital adrenal hyperplasia. Clin Endocrinol 2006;65:45-50.

24 Buttgereit F, Smolen JS, Coogan AN, Cajochen C. Clocking in: chronobiology in rheumatoid arthritis. Nat Rev Rheumatol 2015; 11:349-56.

Address for correspondence: Dr AB Reddy, Wellcome Trust - Medical Research Council Institute of Metabolic Science, University of Cambridge, Addenbrooke's Hospital, Cambridge CB2 0QQ, UK.

Email: areddy@cantab.net 